\title{
Key Exchange Using Passwords and Long Keys
}

\author{
Vladimir Kolesnikov and Charles Rackoff \\ Dept. Comp. Sci., University of Toronto, Toronto, ON, M5S 3G4, Canada \\ $\{$ vlad, rackoff $\} @ c s . u t o r o n t o . c a$
}

\begin{abstract}
We propose a new model for key exchange (KE) based on a combination of different types of keys. In our setting, servers exchange keys with clients, who memorize short passwords and carry (stealable) storage cards containing long (cryptographic) keys. Our setting is a generalization of that of Halevi and Krawczyk [16] (HK), where clients have a password and the public key of the server.

We point out a subtle flaw in the protocols of HK and demonstrate a practical attack on them, resulting in a full password compromise. We give a definition of security of $\mathrm{KE}$ in our (and thus also in the $\mathrm{HK}$ ) setting and discuss many related subtleties. We define and discuss protection against denial of access attacks, which is not possible in any of the previous KE models that use passwords. Finally, we give a very simple and efficient protocol satisfying all our requirements.
\end{abstract}

\section{Introduction}

We consider the goal of enabling multiple independent secure conversations between pairs of parties over an insecure network. The most convenient and natural way to achieve this is to perform a key exchange (KE), that is to provide the parties with matching randomly chosen keys that can be used for securing (only) a particular conversation. Of course, each player wants to communicate with a particular person, and even a powerful adversary $A d v$ should not be able to match him up with a wrong partner. Therefore, players must possess some secret information with which they can authenticate themselves. The kind of information that is available to players determines the setting of KE. The simplest KE setting is when players have a shared random string. $\mathrm{KE}$ is more complicated in the public key setting, where parties have public/private key pairs with the public keys securely published. The most difficult setting is the pure password setting, where parties only have a short (presumably memorizable) shared password. We note that pure password KE protocols, at least in the standard model, are currently rather complicated and inefficient, due to the complexity of the setting.

\section{$1.1 \quad$ Our Setting}

Consider the client-server setting where both long keys and short keys (passwords) are used for KE. Assume that the server's (e.g. bank's) keys are securely stored. We

\footnotetext{
* The full version of this paper, containing a rigorous proof of security, appears in the Eprint archive [17].
} 
take advantage of the inherent logistical differences in how keys are stored by the client (password in memory, long key on a storage card), to achieve more robust security than what is possible by using either type of key alone. Indeed, possession of long keys allows strong security guarantees against an online attacker. However, long keys can not be memorized, and thus must be stored, perhaps on a convenient plastic storage card. This is the vulnerability of this solution - the card may be (relatively) easily stolen by a physical attacker. On the other hand, passwords may be memorized, need not be stored, and thus can not be stolen. However, the protection against an online attacker one can hope to achieve with passwords is rather weak - passwords can always be guessed with relatively high probability. The only (somewhat satisfactory) protection against guessing attacks is recognizing them and refusing connection after a predetermined number of password failures ${ }^{1}$.

Combining the benefits of both settings allows us to obtain a system, secure against both types of attack, and thus suitable for protection of sensitive information. This model is even more appealing due to its wide acceptance - it is natural for us to think of a card and a password, when we do, say, personal banking. More motivation is given in Sect. 3 .

\subsection{Our Contributions}

We demonstrate a dangerous practical attack on the Halevi and Krawczyk (HK) [16] protocols, resulting in full compromise of any client's password (Sect. 2). The elegance, simplicity and practicality of the HK model and protocols resulted in their widespread practical use (e.g. their variants are being considered for parts of the IETF key exchange standard $[12,10])$. Therefore, the discovery of our attack may also have an important practical impact.

We propose and advocate the above Combined Key model of key exchange (ckKE). To the best of our knowledge, it has never been formally discussed. ckKE is a generalization of the HK model.

We give a formal definition of security of ckKE (Sect 3). Defining KE even in simpler settings has proven to be notoriously difficult, with a variety of (only seemingly!) innocuous decisions to be made. We discuss the subtleties of many of our choices, such as the necessity of tightness in the allowed success of the adversary, distinguishing the types of failures and reporting them, etc. Much of our discussion (e.g. on tightness of allowed success of the adversary $A d v$ ) also applies to and benefits pure password models.

We aim to make our definition as simple and natural as possible. For example, we require the server to explicitly indicate in its output whether a password failure occurred. We find this more intuitive than defining password guessing attack as an act of interference by the adversary (e.g. a successful impersonation!), as done in previous formalizations, such as [16,2]. Moreover, in previous formalizations, such as $[16,2,5]$, the attacks are accounted by the environment; the server may not even "know" they occurred (e.g. in case of successful impersonation),

\footnotetext{
${ }^{1}$ We mention (but do not explicitly address) a variation of this defense against "too many" password guessing attacks. There the server limits the rate with which logins can be made, e.g. by exponentially increasing wait times between unsuccessful logins.
} 
which makes attack recognition in practice less intuitive. We also find the game style of definitions (used in this paper) generally simpler and less prone to error than the simulation style (see discussion on the style of definition in Sect. 3.1 for more details).

We discuss unique security features available in ckKE "for free", such as the possibility of protection against the following Denial of Access (DoA) attack. $A d v$, attacking a player $P$, tries to connect to $P$ 's partner $Q$, using any password $p w d$. If $p w d$ is correct, $A d v$ wins; if not, $A d v$ continues until he wins or $Q$ refuses to connect to $P$. Then a legitimate $P$ can no longer connect to $Q$. This easy to mount attack is unavoidable in any password-based setting (including $\mathrm{HK}$ ) and is highly disruptive. We are not aware of the prevention of this attack being previously formalized. We formalize this attack and show how to prevent it in our model.

Finally, we give a very simple and efficient two flow KE protocol and prove its security (Sect. 4). An important feature of our protocol is that its flows are independent of each other, and thus can be sent in any order (or simultaneously), allowing for more flexibility and round efficiency.

\subsection{Related Work}

The problem of key exchange has deservedly received a vast amount of attention (e.g. $[11,3,18,1,20,8,9])$. The more complicated setting of pure password-based $\mathrm{KE}$ (pwKE) was first considered by Bellovin and Merritt [4]. Formal definitions (and protocols) in this setting were given by Bellare, Pointcheval and Rogaway [2], Boyko, Mackenzie and Patel [6], Goldreich and Lindell [13], and, recently, by Canetti et al. [7], as well as by many others.

Most relevant to our work is the problem of password-based KE in the asymmetric client-server setting, where the client has a password and the public key of the server. The question of resistance to off-line password-guessing attacks in this setting was first raised by Gong, et al. [14]. Later, Halevi and Krawczyk [16] formalized the notion of one-way password authentication in this setting and gave very simple and efficient protocols realizing it. They also extended their protocols to achieve key exchange with mutual authentication and perfect forward secrecy. The HK model is much simpler than the pure password model. The work of HK was the inspiration of our paper.

Further, Boyarsky [5] criticised the protocols of the earlier version [15] of [16] and suggested his own formalization of the same model. He showed several ways to amend a variant of protocols of [15] to satisfy his definition. We stress that he does not criticize protocols of the later version [16] we are considering.

Pinkas and Sander [19] consider heuristic approaches to securing passwordonly based authentication. They increase the cost of password-guessing and DoA attacks by using reverse Turing tests (RTT), that is, problems that are easy to solve for humans, but not for computers. We approach a different problem. In particular, RTT techniques can not increase security of a particular client against a determined attacker. 


\section{Attacking the Protocols of Halevi and Krawczyk [16]}

Halevi and Krawczyk give four versions of their protocol (suitable for different tasks: password transmission, one-way authentication, and key exchange in two settings). Three of the four versions (with the exception of the Encrypted Password Transmission protocol) are (similarly) affected. We demonstrate our attack on their key exchange protocol.

The Halevi-Krawczyk protocol. Let $S$ be a server with the public key $p k_{S}$, and $p$ be the password shared between $S$ and the client $C$. Let function $f(\cdot ; \cdot)$ be one-to-one on its components, i.e. for every fixed strings $p, x$, functions $f(p ; \cdot)$ and $f(\cdot ; x)$ are one-to-one. Let $E=(G e n, E n c, D e c)$ be a CCA2 secure encryption scheme.

Construction 1. (The Halevi-Krawczyk Mutual Authentication and Key Exchange Protocol $\left.\left(\Pi_{H K}\right)\right)$

\begin{tabular}{lll}
$S$ & & $C$ \\
\hline pick a nonce $n$ & $n, p k_{S} \rightarrow$ & $\begin{array}{l}\text { verify } p k_{S} \\
\text { pick random long key } k\end{array}$ \\
decrypt and verify & $\leftarrow C, n, E n c_{p k_{S}}(k, f(p ; C, S, k, n))$ & \\
$y:=P R F_{k}(n, S, C)$ & $y \rightarrow$ & check $y=P R F_{k}(n, S, C)$ \\
set $K=P R F_{k}(y)$ & & set $K=P R F_{k}(y)$
\end{tabular}

The "decrypt and verify" step outputs "FAIL" if the encryption is invalid or the received value of $f$ does not match what $S$ computes himself. The nonces must satisfy the only requirement that they never repeat.

Our Attack exploits the structure of $f$. We show that the conditions imposed on $f$ are insufficient. The flaw of the proof of security of the protocol seems to be in the incorrect conclusion in Footnote 9 on p. 258 of [16]. We note that it is possible to make the proof (of security of one-way password authentication protocol) of Halevi and Krawczyk go through by additionally requiring that $f(\cdot ; C, \cdot) \neq f\left(\cdot ; C^{\prime}, \cdot\right)$ for any unequal client names $C, C^{\prime}$.

For simplicity, we describe our attack on a specific instantiation of $\Pi_{H K}$. We stress that natural variants of our attack apply to many choices for $f$, and for nonce strategies, as well as for other parameter settings.

Let client names and passwords be 10 bits long, and nonces be 30 bits long. For a variable $V$, let $v_{i}$ be the $i$-th bit of $V$. For example, $C=\left\langle c_{1}, c_{2}, \ldots, c_{10}\right\rangle$ is the name of the honest player, and $n=\left\langle n_{1}, n_{2}, \ldots, n_{30}\right\rangle$ is the nonce. Let the function be $f(p ; C, S, k, n)=\left\langle c_{1}, \ldots c_{9}, c_{10} \oplus p_{1}, n_{1}, \ldots n_{21}, n_{22} \oplus p_{2}, \ldots, n_{30} \oplus\right.$ $\left.p_{10}, S, k\right\rangle$. Finally, let nonces be chosen sequentially starting from 0 . Note that this is a valid configuration of $\Pi_{H K}$.

The attack proceeds as follows. $A d v$ creates an honest server $S$, an honest client $C$ with any name $C=\left\langle c_{1}, c_{2}, \ldots, c_{10}\right\rangle$, and a bad client $B$ with the name $B=\left\langle c_{1}, c_{2}, \ldots, c_{10} \oplus 1\right\rangle$ and a randomly chosen password $p^{\prime}=\left\langle p_{1}^{\prime}, \ldots, p_{10}^{\prime}\right\rangle$. Let $p$ be $C^{\prime}$ 's password. Suppose for now that $p_{1} \neq p_{1}^{\prime}$, i.e. passwords of $C$ and $B$ differ in the high order bit. $A d v$ observes one execution of KE between $S$ and $C$. $A d v$ 
records the encryption $e$ sent by $C$ and the nonce $n$ (for concreteness, say $n=$ 00..00, e.g. $n$ is the first nonce). Now, $B \operatorname{logs}$ into $S$ as himself, as follows. $S$ sends the nonce $n^{\prime}=n+1=00 . .01$, and $B$ replies with $\left\langle B, n^{\prime}, e\right\rangle$. Now, if $S$ doesn't fail, the password of $C$ is computed as $p w d=\left\langle p_{1}^{\prime} \oplus 1, n_{22} \oplus n_{22}^{\prime} \oplus p_{2}^{\prime}, \ldots, n_{30} \oplus n_{30}^{\prime} \oplus p_{10}^{\prime}\right\rangle$ (since for $i=22, \ldots, 30$, it must be that $n_{i-20} \oplus p_{i}=n_{i-20}^{\prime} \oplus p_{i}^{\prime}$ ). Also, if $p=p w d$, then $S$ must accept, since $f\left(p^{\prime} ; B, S, k, n^{\prime}\right)=f(p w d, C, S, k, n)$. Thus, if $S$ fails, pwd is eliminated from the possible passwords list.

$B$ proceeds logging in as himself another $2^{9}-2$ times, eliminating different passwords one by one, until $S$ accepts and that fact determines $C$ 's password. If $S$ does not accept after $B$ logged in $2^{9}-1$ times, $B$ changes the first bit of his password with the server, and repeats the above entire attack (say, starting with a nonce ending with nine zeros), searching the other half-space. Finally, the two possible unchecked passwords can be verified by the same approach (and changing the password of $B$ ).

We stress that there were no attempts at impersonating $C$ or $S$, and all failures are attributed to $B$. Neither $C$ nor $S$ know that $C$ was attacked, thus $C$ 's account is never blocked. If $B$ 's account is blocked due to failures, $B$ can claim mistyping and restore access. Moreover, there is no need to attack from only $B$ 's account; the attack can be easily distributed to try only a few passwords from each of many bad accounts. Again, it is easy to see that our attack is naturally generalizable to many practical instantiations of $\Pi_{H K}$.

On Boyarsky's [5] amendments of HK. The earlier version [15] of [16] had essentially the same protocol as [16], with the exception of the imposed requirements on the encryption scheme ([15] only required so-called one-ciphertext verification attack resistance, vs ciphertext verification attack resistance in [16]). Boyarsky [5] (independently from the revision resulting in the current version [16]) discovered the insufficiency of the weaker encryption. He gives his own formalization of the model and suggests three different amendments (see Sect. 5 of [5]) of the protocols of [15]. Boyarsky limits his consideration to the case where $f$ is a concatenation function; thus our attack is not applicable to his protocols.

\section{Key Exchange in the Combined Keys Model}

Recall from the discussion in the Introduction that our setting (client carrying a plastic storage card and remembering a password) allows the advantage of robustness, that is graceful degradation of security in case one of the two types of keys is compromised. In particular, if the client's password is compromised, the security of KE should not suffer. On the other hand, if the card is compromised (e.g. copied), the remaining security should be that of the HK password model.

On resistance to server compromise. Halevi and Krawczyk briefly discuss resistance to insider attacks, i.e. attacks by rogue server employees who have access to some, but not all, private information stored on the server (see Sect. 3.3 in [16] for discussion of heuristic defense approaches). As another advantage of our setting, we mention that it allows stronger protection against server com- 
promise. For example, public/private key pairs for each client $C_{i}$ can be set up and used appropriately. Of course, an attacker who steals all the server data would now be able to successfully pose as the server. However, he can be prevented from posing as a client, as long as the client's private key remains secret. We note that such protection will require significant additional complexity of the definition and the protocol, and we leave it outside the scope of this paper. Therefore, as do Halevi and Krawczyk, in our exposition we assume that the server is secure, and his private information is never compromised.

On Denial of Access (DoA) attacks resistance. Recall that in the HK (and also in the pure password) setting, security critically depends on the ability of servers to suspend clients' accounts if there are "too many" password failures. At the same time, it is all too easy for $A d v$ to cause them, making systems unusable by a trivial and easily mounted attack. In our combined key setting, it is natural to introduce protection against such DoA attacks. This can be done by requiring that polytime attackers can not cause password failures (and thus account suspension) without possession of long keys, stored on the card of the client. Of course, $A d v$ may attempt attacks even without having the long keys, and furthermore, such attacks may be noticed by the servers. However, it is not hard to ensure that $A d v$ does not learn anything from such attacks. This can be done, for example, by server first verifying possession of the long key (e.g. in form of a MAC), and immediately failing, if such verification failed. Then $A d v$ does not learn anything about pwd, since it was not even used by the server. Therefore, such password guessing attacks are not a threat, and can be ignored. We formalize resistance to DoA attacks in our definition.

In our view, the main reason for using two types of keys is the two qualitatively different layers of protection against compromise. DoA resistance, although an important bonus, may not alone justify the cost of long key storage and management.

The reader may ask why one can't simply do two KE's in the two relevant models (one with parties sharing long keys, and the HK model) and combine the keys to obtain a KE protocol in our model. There are a number of issues to be addressed there. Firstly, a definition of security has to be given anyway which is the bulk of our work. Secondly, natural ways of combining the two KE protocols (such as establishing a secure session using long keys, and sending the password over it) result in less efficient protocols.

\subsection{Pre-definition Discussion}

We start by briefly recalling the general setting for KE. There is a number of players (in our case, they are divided into two types - clients and servers) who have associated credentials, and pairs of whom may have shared common information. We think of a player as an identity, which may have many instantiations. Whenever a player $P$ wishes to talk to another player $Q$, an instance of $P$ is created with the required credentials passed. Thus an instance can be thought of as a participant of a particular conversation. 
It is convenient to separate the notions of identity and instance for several reasons. Firstly, it is easier to talk about the independence of instances. Independence is highly desirable to avoid maintaining state and worry about communication and synchronization between instances. Secondly, a need often arises to have several channels of communication open between two or more parties simultaneously. Then the notion of instance makes it easier to implement and model concurrent executions of KE by a player.

We do not discuss how a player $P$ knows that he wants to talk to a player $Q$. This may be done as part of previous (possibly insecure) communication, scheduled to happen at some predetermined time, or be requested by a higher level protocol. We give $A d v$ the power to initiate conversations between players to model all possible scenarios.

Our goal is to enable a secure conversation, or session, between the instances of two players. Key exchange provides corresponding pairs of participants with matching keys that can be used for securing their communication. Of course, the keys of honest parties must appear random to the adversary $A d v$, and $A d v$ must not be able to cause instances to match up in an inconsistent way ${ }^{2}$.

To formalize the latter requirement, we need to define the notion of partners - instances who end up having a ( $\mathrm{n}$ intended) conversation. We use session IDs (SID) to partner instances of players. There are several ways of using SID for this purpose, and we choose what we find to be the most natural - requiring each party that output a key to have an additional output sid. The other ways (e.g. requiring sid to be an input to parties, or requiring existence of a partnering function) seem to be less intuitive. We note that many natural protocols can be naturally modified to produce session ids. The sid output is not necessary in real protocols; it is only used for the purpose of defining and analyzing security of KE protocols.

Definition 1. (KE Partners) Let $P$ be a player. We denote by $P_{i}$ the $i$-th instance of $P$. We write $P_{i}^{Q}$ to emphasize that $P_{i}$ intends to do KE with (some instance of) player $Q$. We say that an instance $C_{i}^{S}$ of a client $C$ and an instance $S_{j}^{C}$ of a server $S$ are partners, if they have output the same session id sid.

Note that no two instances are partners when they are created; they may become partners once they've executed their KE protocols. We stress that $P_{i}$ and $P_{i}^{Q}$ refer to the same instance of $P$. We may omit the superscript in $P_{i}^{Q}$, when it is clear from the context.

Mutual authentication (MA) is an assurance that, if $P_{i}^{Q}$ successfully completed and output a key, there must have been a $Q_{j}^{P}$ "communicating" with him. We choose not to require it, because it can be achieved at the cost of two additional "key confirmation" flows (and refreshing the session key). Moreover, $P_{i}^{Q}$ can never be sure that $Q_{j}^{P}$ "is there" anyway, since $Q_{j}^{P}$ may go offline at any time. Note, it is rather common and accepted to not require explicit mutual authentication for these reasons (e.g. [7]). Further, if we required MA, we must

${ }^{2}$ We note that $A d v$ can cause confusion by mismatching instances of players and making them output unrelated keys. We don't regard this as a problem. 
use a special $\perp$ output symbol to denote failure. In our definition we allow $\perp$, but don't insist on its use.

On the notions of attacks and failures. We first note that a special kind of failure - the password failure - must be introduced in our model to allow protection against DoA attacks. Intuitively, if $A d v$ 's attack is such that the act of failure of the server may reveal some information about the client's password, then such failure is a password failure.

A natural approach to define adversary's ability to attack the system is by counting password checking attempts. However, it is less natural to define what an "attempt" is. Indeed, previous works on password-based key exchange (e.g. $[16,2])$ define "attempt" essentially as the act of $A d v$ 's interference with the exchange of messages between two parties. However, it is less clear, for example, whether an act of $A d v$ changing an insignificant bit of a message or an act of successful impersonation is such an attempt. Moreover, previously, the number of attempts was counted not by the server instances (they are not required to "know" whether a password guessing attack occurred), but by the environment.

An important feature of our definition is that servers themselves determine when, whether and what type of failure occurred. This explicates the notion of a failed password attempt, and ensures server's ability to identify a threat and react to it. Therefore, depending on the kind of failure, we allow servers to output either a failure symbol $\perp$, or a password failure symbol $\mathrm{P} \perp$. We count password failures as $\mathrm{P} \perp$ 's reported by the servers, and clients accounts are suspended (to prevent further password guessing) based solely on that information and a predetermined threshold $q$. Therefore, a misidentification of an attack by the server is an omission of the protocol (opening a possibility of either password checking or DoA attacks), and we deem such protocols insecure.

We note that previous definitions, such as those of $[16,2,5]$, can be similarly amended to ensure "explicit authentication" by additionally requiring that the server output $\mathrm{P} \perp$ when he thinks a password attack has occurred. However, as discussed above, it seems to be cleaner to use the server's output as the only criterion for determining whether such an attack took place. Further, to ensure that the server does not misidentify the attacks, his output would need to be incorporated into the definitions, further complicating them.

The use of smart cards vs storage cards is briefly discussed in Sect. 4.

On the style of definition. As mentioned earlier, we prefer the game style of KE definitions in this paper. We find it easier to understand, since the game of the definition naturally corresponds to the actions and abilities of the adversary. We don't seem to need the complexity of simulation style definitions. An exception seems to be the very complex universally composable (UC) definitions, which can model very subtle issues such as password mistyping (see [7] and discussion in Sect 3.3). In addition to their complexity, UC-secure protocols currently are significantly less efficient than protocols in other frameworks. From another point of view, it is highly desirable to have different styles of definitions to discuss their relative strengths and, hopefully, prove equivalence in some settings. 
On modelling the adversary. We consider a powerful $A d v$, who schedules events (such as creation of players and their instances) and controls all communications. This latter is modelled by the parties not sending messages to each other, but giving them to $A d v$ for delivery. $A d v$ is allowed to arbitrarily modify the messages (including dropping and injecting them) and schedule delivery. We allow $A d v$ to create and arbitrarily initialize a polynomial number of accounts for corrupted clients. Note that in this model the actions of corrupt players need not be discussed separately from the actions of $A d v$, since $A d v$ can simulate all their actions. For example, a message sent by a corrupted party can be viewed as a message injected by $A d v$.

Recall, $A d v$ steals either the long key or the password of a client, and attacks one of the several security features of the protocol. We describe the (five) possible settings as games the attacker plays. (These games cover all cases - the cases that are not discussed explicitly are implicitly covered by stronger settings.)

Game $K E_{1}$ models the most complicated setting where $A d v$ stole the long key of the client, and is attacking a server (that is trying to distinguish server's session key from random). This is the only game where $A d v$ can benefit from guessing a password. Thus, in $\mathrm{KE}_{1} A d v$ is allowed a limited number of $\mathrm{P} \perp$ 's.

Game $K E_{2}$ models the setting where $A d v$ stole the long key and the password of the client, but is attacking a client.

Game $K E_{3}$ models the setting where $A d v$ stole only the password of the client, and is attacking a server.

Game DOA models the inability of $A d v$ to cause password failures without stealing the long key.

Game SID models the inability of $A d v$ to cause two honest parties output different session keys, and is included for technical reasons (see discussion before the game's definition in Sect. 3.2 below).

One way to define security is to describe one adversary who, at some point in his attack, decides which of the five games above he really wants to play. However, since $A d v$ 's breaking abilities vary significantly among the games, defining allowed success of $A d v$ in a "combined" game would be unnecessarily complicated. Therefore, we choose to describe five adversaries, each playing the corresponding game. We define the security of ckKE by inability of any of adversaries to win any of these games "too often". We note that it is possible to define the "combined" adversary model carefully, and to prove that any protocol that is secure with respect to the five adversaries would also be secure with respect to one "combined" adversary.

Liveness. Note that protocols may never terminate (e.g. when $A d v$ cuts the communication channels). Instances may also output special failure symbols instead of (sid, key) pairs (e.g. when they detect $A d v$ 's interference). To ensure usability of KE protocols, we disallow these exceptional cases, unless Adv indeed attacks the system. Thus, we require that in the absence of an adversary, when processes communicate as intended, all sessions terminate, and intended partners output the same session id and key. 


\subsection{Formal Definition of Security of Key Exchange in the Combined Keys Model}

Let $n$ be a security parameter, and $m$ be the number of bits in the password. In general, $m$ can be a function of $n$; interesting cases are when $m$ is constant or logarithmic in $n$. WLOG, say, the password domain is $D=\{0,1\}^{m}$. All players ( $A d v$, clients and servers) are p.p.t. machines. Recall, the notion of partnering is defined in Def. 1.

We start by presenting the KE games. Recall, the first game models the setting where $A d v$ obtained the long key of the client, is attacking a server, and is allowed a limited number of $\mathrm{P} \perp$ 's.

Game $\mathbf{K E}_{1}$. The adversary Adv starts by deterministically choosing the active attack threshold $q \in 1 . .|D|$ (based on the security parameter $n$ ) and creating an (honest) server $S$. Adv chooses $S$ 's name; then $S$ 's public and private keys are set up, and only the public key revealed to Adv. Adv then runs the parties by executing steps 1-5 multiple times, in any order:

1. Adv creates an honest client $C$. Adv is allowed to pick any unused name for the client; the client $C$ is registered with $S$, and long key $\ell$ and password pwd are set up and associated with $C$. Only one honest client can be created. Adv is given the long key $\ell$, but not pwd.

2. Adv creates a corrupt client $B^{i}$. Adv is allowed to initialize him in any way, choosing any unused name, long key and password for him.

3. Adv creates an instance $C_{i}$ of the honest client $C . C_{i}$ is given (secretly from $A d v)$ as input: his name $C$, the partner server's name $S$, the public key of $S$, the long key and the password of $C$.

4. Adv creates an instance $S_{j}$ of the honest server $S . S_{j}$ is given (secretly from $A d v)$ as input: his name $S$, the private key of $S$, the partner client's name $\left(C\right.$ or $\left.B^{i}\right)$ and that client's long key and password.

5. Adv delivers a message $m$ to an honest party instance. That instance immediately responds with a reply (by giving it to Adv) and/or terminates and outputs the result (either a (sid,session key) pair or the failure symbol $\perp$ ) according to the protocol. The server instance can additionally output the password failure symbol $P \perp$. If the total number of $P \perp$ for the honest client is equal to the threshold $q$, Adv becomes restricted - he can not deliver messages to any instances $S_{j}^{C}$.

Adv learns the output, with the exception of its session key part. Additionally, at any time Adv may "open" any completed honest instance - then Adv is given the session key output by that instance.

Then Adv asks for a challenge on an instance $S_{j}^{C}$ of the server $S . S_{j}^{C}$, who has been instantiated to talk to the honest client $C$, must have completed and not failed. The challenge is, equiprobably, either the key output by $S_{j}^{C}$ or a random string of the same length. Adv must not have opened $S_{j}^{C}$ or a partner of $S_{j}^{C}$, and is not allowed to do it in the future.

Then Adv continues to run the game as before (execute steps 2-5). Finally, Adv outputs a single bit $b$ which denotes Adv's guess at whether the challenge 
string was random. Adv wins if he makes a correct guess, and loses otherwise. Adv cannot "withdraw" from a challenge, and must produce his guess.

Note the following technicality of $\mathrm{KE}_{1}$. It is possible that $A d v$ may find himself unable to complete the game. This may happen only when he had just caused the $q$-th $\mathrm{P} \perp$ (and hence he is not allowed to deliver messages to servers) and he has no completed instances whom he is allowed to challenge. One way to handle this would be to require $A d v$ flip a coin to determine whether he won or lost. We prefer to simply disallow, by this discussion, such behaviour of $A d v$, since the stalemate can be easily avoided by $A d v$ having a "safety instance" complete before he risks the $q$-th $\mathrm{P} \perp$.

In all other $\mathrm{KE}$ games $\left(\mathrm{KE}_{2}, \mathrm{KE}_{3}\right.$, SID and DOA) below, it is possible (and natural) to require that the knowledge of $p w d$ does not help $A d v$. We thus choose to reveal the password to $A d v$ and remove restrictions on the number of $\mathrm{P} \perp$ 's (thus removing the definition of $q$ ). These games are presented by modifying $\mathrm{KE}_{1}$. All of the above three modifications are included in all games below (and the last two are omitted in individual descriptions for conciseness).

Game $\mathrm{KE}_{2}$ models the setting where $A d v$ stole the long key and the password of the client, but is attacking a client.

Game $\mathbf{K E}_{2}$. This game is identical to $K E_{1}$, with the following additional exceptions.

- Adv is given pwd (in addition to $\ell$ ) and must challenge an honest client instance $C_{i}^{S}$, who is talking to $S$.

Game $\mathrm{KE}_{3}$ models the setting where $A d v$ stole only the password of the client, and is attacking a server.

Game $\mathbf{K E}_{3}$. This game is identical to $K E_{1}$, with the following additional exceptions.

- Adv is given pwd, but not the long key $\ell$.

Game SID enforces a non-triviality condition, preventing parties from improperly partnering up (e.g. by unnecessarily outputting the same session ids). Recall, $A d v$ is not allowed to challenge parties whose partner has been opened, and we need to ensure that $A d v$ is not unfairly restricted.

Game SID. This game is identical to $K E_{1}$, with the following additional exceptions.

- Adv is given pwd (in addition to $\ell$ ) and does not ask for (nor answers) the challenge.

- Adv wins if any two honest partners output different session keys.

Finally, game DOA models resistance to the Denial of Access (DoA) attacks.

Game DOA. This game is identical to $K E_{1}$, with the following additional exceptions. 
- Adv is given pwd, but not the long key $\ell$.

- Adv does not ask for (nor answers) the challenge.

- Adv wins if a server instance $S_{j}^{C}$ outputs $P \perp$.

Definition 2. (Secure Key Exchange in the Combined Keys Model.) We say that a key exchange protocol $\Pi$ is secure in the Combined Keys model, if for every polytime adversaries $A d v_{1}, A d v_{2}, A d v_{3}, A d v_{\text {sid }}$ and $A d v_{\text {doa }}$ playing games $K E_{1}$, $K E_{2}, K E_{3}, S I D$ and DOA, their probabilities of winning (over the randomness used by the adversaries, all players and generation algorithms) is at most only negligibly (in $n$ ) better than:

$-1 / 2+\frac{q}{2|D|}$, for $K E_{1}$,

$-1 / 2$, for $K E_{2}$ and $K E_{3}$,

- 0 , for SID and DOA.

KE definition for the HK setting. We note that Halevi and Krawczyk do not formally define the full notion of $\mathrm{KE}$ in their setting, but concentrate on the oneway password authentication of the client to the server. Because ckKE is a generalization of the HK setting and thanks to the modularity of our presentation, it is not hard to extract the KE definition for the HK setting from Def. 2. The only difference between our and the HK settings is that we additionally allow for the use of the long shared key $\ell$. It turns out that it suffices to remove the games that do not allow $A d v$ to know $\ell$ from Def. 2, to obtain a definition for the HK setting. (Of course, we also need to remove the uses of the long key $\ell$ from the remaining games.) Indeed, it is not hard to verify that the remaining games cover all possible attacks $A d v$ can do in the HK setting. We explicate this definition below.

Definition 3. (Secure Key Exchange in the HK Model.) We say that a key exchange protocol $\Pi$ is secure in the Halevi-Krawczyk, or hybrid, model, if for every polytime adversaries $A d v_{1}, A d v_{2}$ and $A d v_{\text {sid }}$ playing (amended as described above) games $K E_{1}, K E_{2}$ and $S I D$, their probabilities of winning (over the randomness used by the adversaries, all players and generation algorithms) is at most only negligibly (in $n$ ) better than:

$-1 / 2+\frac{q}{2|D|}$, for $K E_{1}$,

$-1 / 2$, for $K E_{2}$,

- 0 , for $S I D$.

We note that although the pre- and post-definition discussion (of Sect. 3.1 and 3.3) discusses the ckKE setting, much of it applies to the HK setting as well.

\subsection{Post-definition Discussion}

On the sufficiency of only one honest server and one honest client. We note that definition of security is not strengthened by allowing $A d v$ to create additional (good or bad) servers or good clients. The reason for this is that we assume independence in the initialization procedures of each pair of identities, and each instance is initialized only with information relevant to its partner. More detail follows. 
Consider an adversary who wishes to attack a particular player - a client $C$ or a server $S$. Suppose we allowed creation of additional good or bad servers. Note that initialization of a client $C$ proceeds independently for servers $S^{1}$ and $S^{2}$, and, further, $C_{i_{1}}^{S^{1}}$ has no information about $C_{i_{2}}^{S^{2}}$, that is not known to $A d v$. Therefore, creating accounts for $C$ with more than one server and instances of $C$ talking to them does not help $A d v$, since it can be simulated by $A d v$. On the other hand, the ability to create many clients with a server is essential, since server instances talking to different clients do share common information among themselves - the secret key of the server. In fact, we exploit that in our attack on $\Pi_{H K}$. Only one honest client is sufficient, however, since additional honest clients can be played by $A d v$. We note that had we allowed clients to possess information common to two or more servers, we would have to allow $A d v$ to create additional bad servers.

Addressing Boyarsky's criticism of the single-user case ([5]), we note that our definition allows $A d v$ to determine whether two honest clients have the same password, causing at least an (expected) one $\mathrm{P} \perp$ on each of the two clients. However, we don't see it as a problem, since, with high probability, clients' passwords differ. Therefore, determining a large clique of users with the same password would cause a large number of system-wide password failures and not cause bigger than expected "bang for the buck".

On the order of creation of good client and revealing the long key $\ell$. $A d v$ should first create the good client, and only then be allowed to see $\ell$. This is the way the attack works in real life. Had we reversed the order, it would be easy to construct good protocols that would be defined insecure (e.g., a server leaks some information, if the client's name is the same as $\ell$.)

On the allowed success of $A d v$ in $\mathbf{K E}_{1}$. Consider the success an adversary can always achieve (and therefore must be allowed in our definition). After $q$ queries, $A d v$ can guess the password with probability $q /|D|$, and if he fails to guess it, he can distinguish the key from random with probability $1 / 2$. Therefore, we should allow $A d v$ 's probability of success of at least $\frac{q}{|D|}+\frac{1}{2} \frac{|D|-q}{|D|}=\frac{1}{2} \frac{q+|D|}{|D|}=$ $\frac{1}{2}+\frac{q}{2|D|}$.

On independence of the states of instances. In our model, there is no global information, and state is not preserved between executions of instances of players. Therefore, for example, it is not possible for an instance to know exactly how many $\mathrm{P} \perp$ 's occurred. Nevertheless, some communication and preservation of state can be achieved with the help of the adversary, as follows. The private key of $S$ now additionally includes an $n$-bit MAC key $k_{M}$. Whenever $S_{j}$ wants to publish a message $m$, he gives $\left(m, M A C_{k_{M}}(m)\right)$ to $A d v$. The server's protocol has an optional field in one of the expected messages. $S_{j}$ only accepts the properly MAC'ed messages in that field (this is essential, so that $A d v$ cannot forge messages). We stress that communication may only happen if it is in the interest of $A d v$. Therefore, it can not be used to increase security of protocols, but mainly to uncover weaknesses of definitions (see example in the next topic). 
On continuing the game after $q \mathbf{P} \perp$ 's. In the real world, at least ideally, after $q \mathrm{P} \perp$ 's, the server knows there is an attack on $C$, and will not accept new connections and will terminate all incomplete instances. How should we model this in our KE games? Although $S$ may have cut communication with $C$, old sessions may still exist, and we need to ensure that they remain secure. That is why we allow the game to continue as before, but disallow sending messages to the server instances after $q \mathrm{P} \perp$ 's occurred.

Observe that once $A d v$ got the challenge, "trying" another password may not help him much. Therefore, in particular, it is crucial to allow to challenge instances after $q \mathrm{P} \perp$ 's occurred.

It is not hard to design a concrete protocol demonstrating the necessity of our choice. Take a secure protocol $\Pi$. Modify it as follows to obtain $\Pi^{\prime}$. Once a $\mathrm{P} \perp$ of an honest client $C$ occurred in the game (see above discussion on independence of states), in all future sessions with instances of $C$ the all-zero session key is chosen with fixed small, but non-negligible probability ( say prob $=\frac{1}{|D|^{3}}$ ). Clearly, this is a bad protocol, since after performing only one active attack, an attacker certainly breaks into one of the next few sessions. However, $\Pi^{\prime}$ would be deemed secure according to the definition, if $A d v$ is not allowed to challenge after $q \mathrm{P} \perp$ 's (this is because $A d v$ is allowed only one challenge, and he does not know which is the weak session. The expected advantage of $A d v$ is less than what he gets from the $q$-th password try.)

On the necessity of tightness in defining the allowed success of $A d v$. Note that for every non-negligible slack allowed in $A d v$ 's success, there is a natural variant of $\Pi^{\prime}$ above, deemed secure by such definition. While one may be tempted to not be very careful in denying $A d v$ "a few extra password tries", $\Pi^{\prime}$ has a much more dangerous vulnerability, which really should be prevented. We remark that in the password-only setting, if an indistinguishability of challenge based security definition does not require tightness, a simpler variant of $\Pi^{\prime}$, where players always output an all zero key with sufficiently small (yet nonnegligible) probability, would be deemed secure.

On clients mistyping the passwords. How should we model the case when an honest client mistypes the password and causes $\mathrm{P} \perp$ ? Consider the following protocol. Take a secure protocol, and modify it, so that $S_{j}^{C}$ reveals $\ell$ once $\mathrm{P} \perp$ occurred. It is easy to see that the new protocol remains secure in our definition, since we implicitly assume that $C$ never mistypes the password. Indeed, in our definition, if a $\mathrm{P} \perp$ occurred, it must have been caused by $A d v$. Since $A d v$ cannot cause $\mathrm{P} \perp$ without possession of $\ell$, it is $\mathrm{OK}$ if $S_{j}^{C}$ reveals $\ell$. However, intuitively, we would not want to call such a protocol secure.

The only way to formally address the issue in our model is to allow $C$ to mistype the password. A natural first idea is to allow $A d v$ to instantiate clients with the password of his choice. However, it is not clear that this models real life - most often clients mistype their passwords to something related. Further, this would not address the protocol that reveals $\ell$ if the $p w d$ is mistyped as $p w d+1$, or, more generally, if the $p w d$ is mistyped as a function of $p w d$. 
A natural next idea is to instantiate clients with the password being $f(p w d)$, where the deterministic function $f$ is specified by $A d v$. Only such an $f$ that does not allow to check more than one password at a time may be allowed, and therefore strong restrictions on $f$ are necessary. Indeed, setting $f(p w d)=0$ on the first half of password domain $D$, and $f(p w d)=p w d$ on the second half, allows $A d v$ to check half of password domain in one try. Restricting $f$ to be a permutation does not work either, since applying such $f$ allows to check whether pwd is a fixed point of $f$. Therefore functions $f$ that have more than one or fewer than $|D|-1$ fixed points are not allowed. At the same time, it is not hard to see that functions with $0,1,|D|-1$ or $|D|$ fixed points do not allow $A d v$ to check more than one password at a time when server is running a secure protocol, and thus may be allowed in our definition. Indeed, a function with 0 fixed points always causes $S_{j}^{C}$ to $\mathrm{P} \perp$; one with 1 fixed point $f p$ always causes $\mathrm{P} \perp$, unless $p=p w d$, and thus allows to check precisely one password; one with $|D|$ fixed points (identity) never causes $\mathrm{P} \perp$; one with $|D|-1$ fixed points always succeeds, unless pwd is the non-fixed point, and thus allows to check precisely one password.

At the same time, the most natural mistyping functions (e.g. confusing the order of digits) do not satisfy the requirements on $f$ and do help the adversary (e.g. Adv can quickly test if the pin consists of the same decimal digits). More generally, $A d v$ may infer a lot from simply observing a large volume of traffic, noting the patterns of honest clients mistyping their passwords, and matching them with expected patterns. However, it is not clear how to analyze this advantage, so we choose not to include password mistypes in our model at all, with the understanding that protocol designers take this discussion into account.

This subtlety also arises in KE in the pure password model, when passwords need not be chosen uniformly from $D$. Indeed, let $D_{1} \subset D$ be all elements of $D$ that end with a 0 , and $p w d \in D$ is chosen uniformly from $D_{1}$. Then a protocol $\Pi$ that reveals $p w d$ iff $p w d$ is mistyped only in the last digit, would be secure under a natural definition that does not allow mistyping. This is because $p w d$ would not be revealed, unless $A d v$ already had tried it. At the same time, such protocol $\Pi$ should not be deemed secure. We note that the recent definition of password based KE in the complex Universal Composability model ([7]) addresses the issue of mistyping by allowing the environment both choose and type passwords.

On reporting failures to $A d v$ immediately after failing. Consider a modification of $\Pi_{H K}$, where, upon a password failure, the server does not report it to $A d v$, but produces a random key and simulates successful completion of KE. This change would have prevented our attack of Sect 2. However, the achieved security would be illusory, since, in practice, it is hard to simulate successful completion well. Indeed, the fact of $\mathrm{P} \perp$ must be somehow registered and used by $S$. This changes the state of $S$ (in particular, the counter of active attacks is incremented). Since $C$ can login after $q-1$, but not after $q \mathrm{P} \perp$ 's, $A d v$ is able to infer some information about $S^{\prime}$ outputs. To account for such "side channels", we require that players don't have private failure outputs (either $\perp$ or $\mathrm{P} \perp$ ), and $A d v$ is informed of failure as soon as it output. Note that this discussion relates 
to the Additional discussion in Sect. 2.1 of [7], where the authors argue that $A d v$ need not know whether the passwords of two honest partners matched.

To further illustrate this point, suppose $S_{j}$ at some point "knows" he is going to output $\mathrm{P} \perp$, that is, $S_{j}$ entered a state from which all execution paths lead to outputting $\mathrm{P} \perp$, and $A d v$ learned this fact. Suppose $S_{j}$ does not terminate yet, but is waiting to receive another message. Then $A d v$ can delay the delivery of the message indefinitely, $S_{j}$ would never report $\mathrm{P} \perp$, and we don't count it. In particular, adding an extra round of communication to a secure protocol $\Pi$, in which parties say whether they failed, makes $\Pi$ insecure. This is consistent with our desire to force a server to correctly and timely report active attacks.

\section{Our Protocol}

Let $n$ be a security parameter. To simplify discussion, we present our constructions with the domains and ranges of PRFG and MAC equal to $\{0,1\}^{n}$. Let $E=($ Gen, Enc, Dec) be a CCA2 secure public key encryption scheme, $F$ : $\{0,1\}^{n} \times\{0,1\}^{n} \mapsto\{0,1\}^{n}$ be a PRFG, and $M A C:\{0,1\}^{n} \times\{0,1\}^{*} \mapsto\{0,1\}^{n}$ be a message authentication code. Let $N_{C}$ be the name of the client $C$, drawn from $\{0,1\}^{n}$. Shorter names can be used for efficiency, if desired.

Consider the following KE protocol $\Pi$, with two types of players, a server $S$ and a client $C$ who have secretly agreed on a password $p w d \in_{R} D$, a long secret key $\ell \in_{R}\{0,1\}^{n}$. Also, $S$ has generated public/private key pair $\left(p k_{S}, s k_{S}\right)$, and gave $p k_{S}$ to $C$.

Construction 2. (KE in the Combined Key Model (П).)

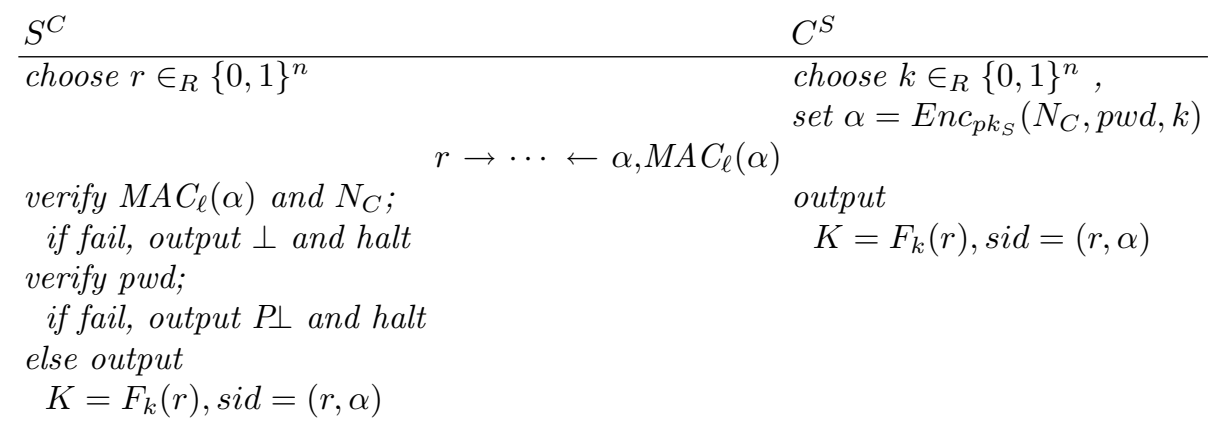

WLOG, we assume that all protocol messages are formed properly (i.e. values are drawn from the appropriate domains, etc.). Then a client instance never fails, while a server instance may. Note that $A d v$ may cause non-partnered parties to output unrelated keys. This is not a problem (see Sect. 3.1 and Footnote 2).

We stress that the two flows of the protocol are independent, and thus either of the parties can be the initiator. The DoA attacks are prevented if $A d v$ does not have $\ell$, even though, in particular, $A d v$ is able to resend old messages of the client. The latter causes a server to output a random (from the point of view of $A d v)$ session key, thus $A d v$ is not able to take advantage of it. This also does 
not enable $A d v$ to "reset" the fail counter in real executions (and thus try many passwords undetected), since the same effect can be achieved by $A d v$ executing a KE between honest $S_{j}^{C}$ and $C_{i}^{S}$, and then cutting the communication.

We treat the policies of account suspension and resetting of failure counters as external to our discussion, but stress that care should be taken in designing and implementing them. In particular, the client's explicit consent (communicated over a secure session) should be necessary for resetting the failed attempts counter, since otherwise $A d v$ can be undetected when trying passwords between legitimate client logins. A natural scenario would be that the server asks the client whether he mistyped the password a certain number of times, and when client confirms, the fail counter is reset.

We further note that we can prevent $A d v$ from resending $C$ 's old replies to $S$ (e.g. if it is undesirable to have "hanging" sessions) by including $r$ in the encryption of the client's reply and adding the corresponding verification step to $S$. We chose not to include it because it disallows the independence of flows of KE, and it is unclear whether hanging sessions are "worse" than hanging KE.

An alert reader will notice that smart cards may be gainfully used in place of client's storage cards. A smart card may hide the long key $\ell$, only exposing the MAC'ing interface. An interesting setting is when $A d v$ can "borrow" and return (but not copy) the card, obtaining only a period of ability to MAC strings of his choice. Our protocol will not benefit from such security improvements: $C$ 's messages are independent of $S$ 's, and thus $A d v$ can MAC all the strings he might possibly need for an attack (e.g. strings containing all possible passwords) in one batch. Again, including $r$ in the encryption of $C$ 's reply resolves this problem.

$\Pi$ is secure. We first observe that for every $A d v_{\text {sid }}$ and $A d v_{d o a}$ playing games SID and DOA, their probability of winning is negligible. Indeed, in our protocol, partners never output different keys (since the session key is determined by sid). As for $A d v_{d o a}$, for a server to output $\mathrm{P} \perp$, it is necessary to forge a MAC on an encryption not produced by any of the honest clients. This is only possible with negligible probability without the knowledge of the long key $\ell$, assuming security of MAC.

Due to the lack of space, we formally consider the remaining games $\mathrm{KE}_{i}$ and adversaries in the full version [17], which appears in the Eprint archive. The structure of our proof is as follows. We start by reducing the KE adversaries to ones playing much simpler games. As a second step, we show that existence of new adversaries implies insecurity of either of the employed primitives. To give a flavor of the proof within the limited space, we include the most interesting intermediate game in Appendix A. Altogether, we've proven

Theorem 1. The protocol $\Pi$ of Constr. 2 is a secure key exchange protocol in the combined keys model.

On generalizing Constr. 2. Consider creating a family of protocols parameterized by a function $f$ similarly to the approach of Halevi and Krawczyk. The goal is to shorten the plaintext of the encryption $\alpha$ sent by $C$, which may improve the performance of the protocol. We note that we already reduce the amount of data under the CCA2-secure encryption - it is smaller than in any member 
of the HK families of KE protocols (but note that HK KE additionally achieve mutual authentication). We do not see how to further significantly increase efficiency by applying the HK idea to our protocols.

KE protocols for the HK setting. It is easy to see that removing the uses of the long key $\ell$ from the protocol of Constr. 2 casts it into the HK setting. The obtained protocol (explicated in Constr. 3 below) is a secure KE protocol in the HK setting, according to Def. 3. This conclusion immediately follows from the method of construction and Theorem 1 .

Construction 3. (KE in the HK setting.)

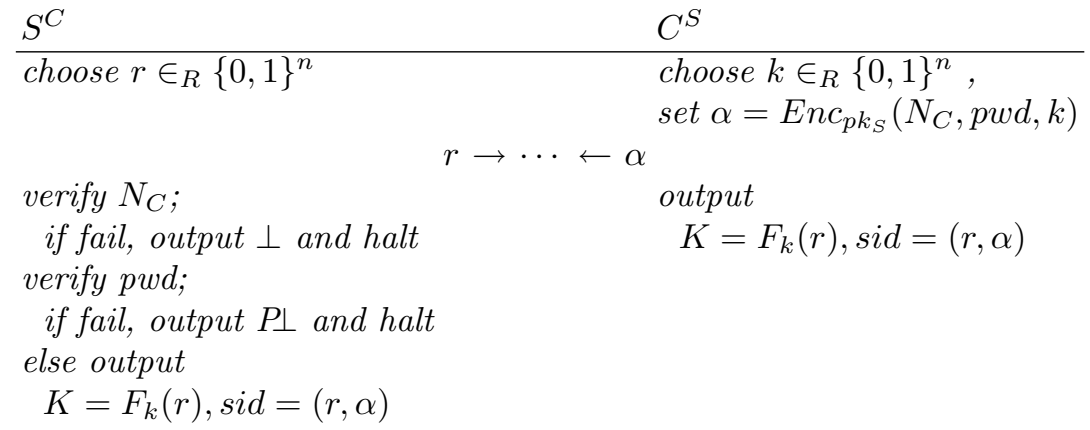

Acknowledgements. We thank Shai Halevi and the anonymous referees of TCC 2006 for very helpful comments on earlier versions of this work. We also thank Ian F. Blake for several stimulating discussions. The authors were in part supported by Natural Sciences and Engineering Research Council of Canada (NSERC) grants. The first author was also supported by Ontario Graduate Scholarship (OGS).

\section{References}

1. Mihir Bellare, Ran Canetti, and Hugo Krawczyk. A modular approach to the design and analysis of authentication and key exchange protocols (extended abstract). In STOC '98: Proceedings of the thirtieth annual ACM symposium on Theory of computing, pages 419-428, New York, NY, USA, 1998. ACM Press.

2. Mihir Bellare, David Pointcheval, and Phillip Rogaway. Authenticated key exchange secure against dictionary attacks. In EUROCRYPT 2000, pages 139-155, 2000 .

3. Mihir Bellare and Phillip Rogaway. Entity authentication and key distribution. In CRYPTO '93: Proceedings of the 13th annual international cryptology conference on Advances in cryptology, pages 232-249, New York, NY, USA, 1994. SpringerVerlag New York, Inc.

4. Steven M. Bellovin and Michael Merritt. Encrypted key exchange: Password-based protocols secureagainst dictionary attacks. In SP '92: Proceedings of the 1992 IEEE Symposium on Security and Privacy, page 72, Washington, DC, USA, 1992. IEEE Computer Society. 
5. Maurizio Kliban Boyarsky. Public-key cryptography and password protocols: the multi-user case. In CCS '99: Proceedings of the 6th ACM conference on Computer and communications security, pages 63-72, New York, NY, USA, 1999. ACM Press.

6. V. Boyko, P. MacKenzie, and S. Patel. Provably Secure Password-Authenticated Key Exchange Using Diffie-hellman. In B. Preneel, editor, Proceedings EUROCRYPT 2000, pages 156-171, 2000.

7. Ran Canetti, Shai Halevi, Jonathan Katz, Yehuda Lindell, and Philip D. MacKenzie. Universally composable password-based key exchange. In EUROCRYPT 2005, pages 404-421, 2005.

8. Ran Canetti and Hugo Krawczyk. Analysis of key-exchange protocols and their use for building secure channels. In EUROCRYPT '01: Proceedings of the International Conference on the Theory and Application of Cryptographic Techniques, pages 453 474, London, UK, 2001. Springer-Verlag.

9. Ran Canetti and Hugo Krawczyk. Universally composable notions of key exchange and secure channels. In EUROCRYPT '02: Proceedings of the International Conference on the Theory and Applications of Cryptographic Techniques, pages 337351, London, UK, 2002. Springer-Verlag.

10. T. Clancy. Eap password authenticated exchange, draft archive. http://www.cs.umd.edu/ clancy/eap-pax/, 2005.

11. Whitfield Diffie and Martin E. Hellman. New directions in cryptography. IEEE Transactions on Information Theory, IT-22(6):644-654, 1976.

12. Internet Engineering Task Force. Eap password authenticated exchange. http://www.ietf.org/internet-drafts/draft-clancy-eap-pax-03.txt, 2005.

13. Oded Goldreich and Yehuda Lindell. Session-key generation using human passwords only. In CRYPTO '01: Proceedings of the 21st Annual International Cryptology Conference on Advances in Cryptology, pages 408-432, London, UK, 2001. Springer-Verlag.

14. L. Gong, M. A. Lomas, R. M. Needham, and J. H. Saltzer. Protecting poorly chosen secrets from guessing attacks. IEEE Journal on Selected Areas in Communications, 11(5):648-656, 1993.

15. Shai Halevi and Hugo Krawczyk. Public-key cryptography and password protocols. In CCS '98: Proceedings of the 5th ACM conference on Computer and communications security, pages 122-131, New York, NY, USA, 1998. ACM Press.

16. Shai Halevi and Hugo Krawczyk. Public-key cryptography and password protocols. ACM Trans. Inf. Syst. Secur., 2(3):230-268, 1999.

17. Vladimir Kolesnikov and Charles Rackoff. Key exchange using passwords and long keys. Manuscript, available from Eprint archive, http://eprint.iacr.org.

18. H. Krawczyk. Skeme: a versatile secure key exchange mechanism for internet. In SNDSS '96: Proceedings of the 1996 Symposium on Network and Distributed System Security (SNDSS '96), page 114, Washington, DC, USA, 1996. IEEE Computer Society.

19. Benny Pinkas and Tomas Sander. Securing passwords against dictionary attacks. In CCS '02: Proceedings of the 9th ACM conference on Computer and communications security, pages 161-170, New York, NY, USA, 2002. ACM Press.

20. Victor Shoup. On formal models for secure key exchange. Technical Report RZ 3120 (\#93166), 1999. 


\section{A An Intermediate Game in the Proof of Theorem 1}

We include the following game $G_{1}$ as an important intermediate step that gives the flavor of the proof of the most subtle case - the inability of $A d v$ playing $\mathrm{KE}_{1}$ win "too often". In the proof (included in the full version [17]) we show that $A d v$ winning $\mathrm{KE}_{1}$ implies an adversary Dist ${ }_{1}$ winning $G_{1}$. We then show that Dist $_{1}$ winning "too often" implies insecurity of one of the employed primitives. Let $n$ be a security parameter.

Game $G_{1}$. A maximum number of "password tries" $q$ is deterministically (based on $n$ ) chosen by Dist ${ }_{1}$ and fixed. The game initializes a CCA2 secure encryption scheme (by generating public and private keys $p k_{S}$ and $s k_{S}$ ) and randomly chooses the password pwd $\in_{R} D$. Only the public key $p k_{S}$ is given to Dist . $_{\text {. }}$. Dist $_{1}$ queries the decryption oracle $O_{D}\left(e^{\prime}\right)=\operatorname{Dec}_{s k_{S}}\left(e^{\prime}\right)$ to obtain decryptions of chosen strings. Then Dist ${ }_{1}$ chooses a "client name" $N_{C}$. Then, for $i=1, \ldots, u$, Dist ${ }_{1}$ queries the encryption oracle $O_{E}$ that produces random encryptions $e_{i}=$ Enc $_{p k_{S}}\left(N_{C}\right.$, pwd, $\left.k_{i}\right)$, where $k_{i} \in_{R}\{0,1\}^{n}$ are chosen randomly

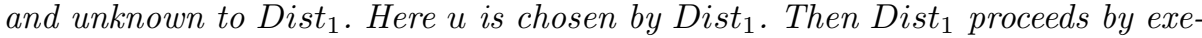
cuting Steps 1 - 2 multiple times, in any order:

1. Dist D $_{1}$ queries the PRFG oracle $O_{F}(i, r)=F_{k_{i}}(r)$, where $k_{i}$ was chosen (but not revealed) by $O_{E}$ during it's $i$-th query. Here $r \in\{0,1\}^{n}$ and $i \in\{1 . . u\}$ are chosen by Dist $_{1}$.

2. Dist ${ }_{1}$ queries the decryption oracle $O_{D}\left(e^{\prime}\right)$, where $e^{\prime}$ is chosen by Dist che $_{1}$. He is not allowed to query $O_{D}$ on any $e_{i}$ obtained from $O_{E}$.

Then Dist chooses $\in\{1, \ldots, u\}$ and $r_{0} \in\{0,1\}^{n}$ and queries the challenge oracle $O_{C}\left(i, r_{0}\right) . O_{C}$ produces a challenge as follows: it randomly chooses a bit $b$ and a string $\rho \in \in_{R}\{0,1\}^{n}$. Then $O_{C}\left(i, r_{0}\right)=F_{k_{i}}\left(r_{0}\right)$ if $b=0$, and $O_{C}\left(i, r_{0}\right)=\rho$ if $b=1$. Dist $t_{1}$ is not allowed to query $O_{C}\left(i, r_{0}\right)$, if he queried $O_{F}\left(i, r_{0}\right)$.

Then, Dist ${ }_{1}$ continues running Steps 1-2, with the exception that he is not allowed to query $O_{F}\left(i, r_{0}\right)$.

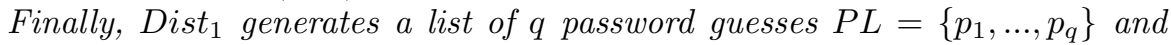
outputs a bit $b^{\prime}$. Dist ${ }_{1}$ wins if $p w d \in P L$ or if $b=b^{\prime}$. 